\title{
Strontium-89 Therapy for Breast Cancer Bone Metastases: Pain Relief Effects and Predictive Factors
}

\author{
Yukinori Okada', Tatsuyuki Abe ${ }^{1}$, Mio Shinozaki', Shinjiro Sakaino' ${ }^{1}$, Hiromichi Gomi', \\ Yasuo Nakajima1, Yasuyuki Kojima ${ }^{2}$, Tomoko Uejima ${ }^{2}$, Koichiro Tsugawa ${ }^{2}$ \\ ${ }^{1}$ Department of Radiology, St. Marianna University School of Medicine, Kawasaki, Japan \\ ${ }^{2}$ Department of Breast and Endocrine Surgery, St. Marianna University School of Medicine, Kawasaki, Japan \\ Email: igaueno512@yahoo.co.jp
}

How to cite this paper: Okada, Y., Abe, T., Shinozaki, M., Sakaino, S., Gomi, H., Nakajima, Y., Kojima, Y., Uejima, T. and Tsugawa, K. (2018) Strontium-89 Therapy for Breast Cancer Bone Metastases: Pain Relief Effects and Predictive Factors. Advances in Breast Cancer Research, 7, 250-257.

https://doi.org/10.4236/abcr.2018.74016

Received: September 1, 2018

Accepted: October 7, 2018

Published: October 10, 2018

Copyright $\odot 2018$ by authors and Scientific Research Publishing Inc. This work is licensed under the Creative Commons Attribution International License (CC BY 4.0).

http://creativecommons.org/licenses/by/4.0/

\begin{abstract}
In this study, we evaluate the analgesic effect of strontium-89 (Sr-89) for patients with breast cancer having multiple bone metastases and identify positive predictive factors. We retrospectively evaluated 15 patients who were administered $\mathrm{Sr}-89$ for painful bone metastases from breast cancer at St. Marianna University Hospital between January 2010 and April 2014. For patients receiving multiple doses, only the first dose was evaluated. Pain relief was defined as a decrease in the score of the Numeric Rating Scale (NRS) or requirement of reduced doses of pain medication 1 - 2 months after being on Sr-89 administration. The associations between pain relief owing to Sr-89 and that owing to bone scan index, interval from bone metastasis onset to Sr-89 administration, zoledronate and denosumab treatment history, hormone therapy/aromatase inhibitor history, and chemotherapy history were assessed. A logistic model was used for statistical analysis. Pain relief was observed in $11(73.3 \%)$ of 15 patients. No statistically significant value was observed between pain relief and bone scan index, interval from bone metastasis onset to Sr-89 administration, zoledronate and denosumab treatment history, hormone therapy/aromatase inhibitor history, and chemotherapy. Thus, Sr-89 was effective for $70 \%$ of patients with breast cancer bone metastases, although positive predictive factors for pain relief could not be determined.
\end{abstract}

\section{Keywords}

Breast Cancer, 89-Sr, Analgesia

\section{Introduction}

The incidence of bone metastases is particularly high in patients with hor- 
mone-dependent cancers such as breast and prostate cancers. Drug therapy with nonsteroidal antiinflammatory drugs (NSAIDs) and narcotics is often used to relieve pain. Zoledronic acid and denosumab are often concomitantly used for delaying the onset of skeletal complications such as increased pain, pathologic fractures, and spinal cord compression. Because external beam radiation provides relief from bone metastasis-induced pain, it is frequently selected for cases in which pain relief from drug therapy is insufficient. External beam radiation is often challenging in cases of multiple bone metastases because the treatment is localized. In November 2007, the use of strontium-89 (Sr-89) as an unsealed source therapy for relief of painful bone metastases was approved in Japan. When Sr-89 is intravenously administered, it is taken up by all sites of abnormal bone turnover. Thus, unlike external beam radiation, a single intravenous dose results in irradiation of multiple bone metastases throughout the skeleton.

However, in breast cancer with bone metastasis, the pain relief rate and predictive factors remain unknown. Therefore, we aimed to evaluate the analgesic effect of $\mathrm{Sr}-89$ in patients with bone metastases from breast cancer and assess the presence of any pain relief predictive factors.

\section{Patients and Methods}

\subsection{Patients}

We reviewed the radiological information system and medical history charts of breast cancer patients who received Sr-89 for painful bone metastases from breast cancer at St. Marianna University Hospital between April 2008 and May 2014 and included 23 patients.

However, 8 breast cancer patients who received Sr-89 for pain relief were excluded because their pain control evaluations were insufficient. Finally, the study population comprised 15 patients who received $\mathrm{Sr}-89$ for painful bone metastases from breast cancer at St. Marianna University Hospital between January 2010 and April 2014. This study was approved by the ethics committee of St. Marianna University School of Medicine (No. 2757).

Sr-89 was administered three times to one patient; however, data only from the first administration were included in the current analysis. All 15 patients were women, and their median age at the time of Sr-89 administration was 58 range, 30 - 73) years. 7 patients were Luminal type, 5 patients were HER2 type, and 3 patients were triple negative type. At the time of Sr-89 administration, nine patients were concomitantly taking narcotics (oxycodone by 7 patients and fentanyl citrate by 2). Six patients were using NSAIDs only.

Using the electronic medical record system, we recorded data such as pain levels before Sr-89 and bone scan index (BSI); duration from bone metastasis onset; diagnosis based on PET-CT, bone scintigraphy, CT, or MRI until Sr-89 administration; use of zoledronate or denosumab; presence/absence of hormone/ aromatase inhibitor therapy; and presence/absence of concomitant chemotherapy. 


\subsection{Evaluation of the Analgesic Effect of Sr-89}

Patients exhibiting improvement according to the Numeric Rating Scale (NRS) or reduced dosage or reduced frequency of painkiller use (NSAIDs, narcotics) 1 - 2 months after being on Sr-89 administration were defined as those experiencing analgesia.

\subsection{Bone Scan Index}

Before Sr-89 administration, bone scintigraphy was necessary. Approximately 2.5 - $3.5 \mathrm{~h}$ after administering 370 - $925 \mathrm{MBq} 99 \mathrm{mTc}-\mathrm{MDP}$, whole-body anterior and posterior images and localized images were acquired using a scintillation camera (ECAM or GXA-7200; Cannon Medical System: Ohtawara Japan). ECAM had a capture rate of $18 \mathrm{~cm} / \mathrm{min}$ and a matrix size of $256 \times 1024$, and GCA-7200 had a capture rate of $17.5 \mathrm{~cm} / \mathrm{min}$ and a matrix size of $256 \times 1024$. We used BONE NAVI version 1 (FUJIFILMRI pharma, Co. Ltd. Tokyo Japan) for bone scintigraphy analysis. Bone scan index (BSI) was calculated as the percentage of weight of summed abnormal hot spots to the entire skeleton. We used BONE NAVI version 1 to calculate BSI automatically. In 15 patients undergoing $99 \mathrm{mTc}-\mathrm{MDP}$ bone scintigraphy before Sr-89 administration, BSI was calculated using BONE NAVI version 1. The median value of BSI was 3.038 (range 0.22 12.265).

\subsection{Duration from Bone Metastasis Diagnosis Onset until Sr-89 Administration}

The median duration from bone metastasis onset until Sr-89 administration was $13(0$ - 51) months.

\subsection{Zoledronate and Denosumab}

At the time of Sr-89 administration, zoledronate was being used concomitantly by 10 patients and denosumab by 4 .

\subsection{Hormonal Agents}

2 patients were receiving progesterone, 6 were being treated with aromatase inhibitors, and 1 was receiving exemestane.

\subsection{Chemotherapeutic Agents}

Chemotherapeutic agents were used concomitantly for 8 patients (S-1 for 1 patient, capecitabine for 1 , doxifluridine + cyclophosphamide for 1 , docetaxel + trastuzumab for 1 , trastuzumab for 3 , trastuzumab + pertuzumab for $1)$.

\subsection{Adverse Events}

Adverse events owing to Sr-89 were evaluated using the National Cancer Institute's Common Terminology Criteria (CTCAE V3.0). 


\subsection{Statistical Analysis}

We used a logistic model to assess pain relief. The significance level was set as $\leq 5 \%$. Statistical processing was performed using the R software.

\section{Results}

The patient's characteristics are given in Table 1.

\subsection{Analgesia}

Significant analgesia was observed in $11(73.7 \%)$ of 15 patients. The dosage of painkillers (including the frequency of rescue) was observed in 5 patients (33.3\%), and improvement in NRS was observed in 6 patients (40.0\%).

\subsection{BSI}

The relationship between BSI and pain relief owing to Sr-89 was not statically significant (odds ratio: 0.869, 95\% CI: $0.695-1.150, \mathrm{p}=0.322$ ).

\subsection{Duration from Bone Metastasis Diagnosis Onset until Sr-89 Administration}

In 1 patient, bone metastasis diagnosis onset until Sr-89 administration was 0 month, and in 2 patients, bone metastasis diagnosis onset until Sr-89 administration was 1 month. These patients received Sr-89 in early stage of bone metastasis. In 12 patients, one metastasis diagnosis onset until Sr-89 administration was 5 to 51 months. The relationship between duration from bone metastasis diagnosis onset until Sr-89 administration was not statically significant (odds ratio: $0.958,95 \%$ CI: $0.879-1.040, \mathrm{p}=0.329$ ).

Table 1. Patients characteristics.

\begin{tabular}{cc}
\hline Number of patients & 15 \\
Sex & All females \\
Age (years) & Median 58 (30 - 73) \\
subtype & Luminal: 7 \\
BSI (Bone Scan Index) & HER2: 5 \\
Duration from bone metastasis diagnosis & Triple negative:3 \\
onset until Sr-89 administration (months) & Median 3.028 (0.22 - 12.265) \\
Zoledronate and denosumab & Median $23(0-51)$ \\
& Zoledronate: $10(66.7 \%)$ \\
Hormonal agents & Denosumab: $4(26.7 \%)$ \\
& No: $1(6.6 \%)$ \\
& Yes: $9(60 \%)$ \\
& No: $6(40 \%)$
\end{tabular}




\subsection{Zoledronate and Denosumab}

In 10 patients, zoledronic acid and $\mathrm{Sr}-89$ was used in combination and significant analgesia was observed in $6(60.0 \%)$ patients. In 4 patients, denosumab and Sr-89 was used in combination and significant analgesia was observed in 4 (100.0\%) patients. The relationship between zoledronic acid or denosumab and pain relief owing to $\mathrm{Sr}-89$ was not statically significant (odds ratio: $1.330,95 \%$ CI: $0.089-20.70, \mathrm{p}=0.837$ ).

\subsection{Hormonal Agents}

In 9 patients, hormonal agents and $\mathrm{Sr}-89$ was used in combination and significant analgesia was observed in 7 (77.8\%) patients. In 6 patients, hormonal agents and $\mathrm{Sr}-89$ was not used in combination and significant analgesia was observed in $4(66.7 \%)$ patients. The relationship between hormonal agents and pain relief owing to $\mathrm{Sr}-89$ was not statically significant (odds ratio: 1.750, 95\% CI: 0.173 $17.70, \mathrm{p}=0.635)$.

\subsection{Chemotherapeutic Agents}

In 8 patients, chemotherapeutic agents and Sr-89 was used in combination and significant analgesia was observed in $5(62.5 \%)$ patients. In 7 patients, chemotherapeutic agents and $\mathrm{Sr}-89$ was not used in combination and significant analgesia was observed in $6(75.0 \%)$ patients. The relationship between chemotherapy and pain relief owing to $\mathrm{Sr}-89$ was not statically significant (odds ratio: 0.278, 95\% CI: $0.021-3.58, \mathrm{p}=0.326)$.

\section{Discussion}

In this study, we found that Sr-89 improve the bone metastatic pain in $70 \%$ patients with breast cancer bone metastases, although we were unable to determine positive predictive factors for pain relief.

For breast cancer patients with bone metastases who do not achieve enough pain relief from pain medication, external beam radiation is frequently selected. However, a limitation of external beam radiation is its purely local effect, rendering it ineffective if there are multiple indistinct painful sites. After pain at the most intense site is improved by external beam radiation, pain is then frequently experienced at other locations, requiring multiple external beam treatments. External beam therapy is contraindicated when interstitial pneumonia is present in the radiation field [1]. In the vertebral body, once radiation has been performed, the amount of radiation to the spine is limited by the total dose to the spinal cord. In some cases, re radiation to the spine is reported [2]. But, the safety of re radiation to the spine is not established. The mean range of $\beta$ particles emitted by $\mathrm{Sr}-89$ is approximately $2.4 \mathrm{~mm}$. If $\mathrm{Sr}-89$ is accumulated to the bone metastases in the thorax, the lung irradiation dose is very little. As the mean range of Sr-89 $\beta$ particles are short and cannot reach the lung from the bone metastases in the thorax. So, in patients with bone metastasis in the thorax and internal 
pneumonia, Sr-89 can be used in safety. Similarly, when Sr-89 is accumulated to the bone metastases in the vertebral lesion, the spinal cord irradiation dose is little. Unlike external beam therapy, which is usually fractionated over days or weeks, $\mathrm{Sr}-89$ is administered as a single intravenous injection. Baziotis et al. reported that in 64 metastatic breast cancer patients, the rate of complete or partial disappearance of bone pain was $81 \%$ [3]. Fuster et al. reported a $92 \%$ rate of complete or partial disappearance of pain in 40 breast cancer patients [4].

Although comparison is difficult owing to different quantitative pain assessment methods being used, pain relief was observed by either reduced doses of painkillers or lower NRS in 11 of 15 patients (73.3\%), a result similar to that of Baziotis et al. and Fuster et al.

Various reports predicting the pain-relieving capabilities of Sr-89 have been published. Dafermou et al. compared osteoblastic and mixed bone metastases by radiography and found that pain relief was observed in $61.6 \%$ of cases of osteoblastic bone metastases and $62.5 \%$ of cases of mixed metastases, whereas the pain relief effect was just $42.9 \%$ in osteolytic bone metastases. Although there was not significant difference $(P=0.07)$, the results show that the pain relief effect tended to be diminished in osteolytic bone sites [5].

Kraeber reported that in 94 cases of prostate cancer metastasis to the bone, pain relief owing to Sr-89 was observed at about the same rate in cases with 10 or fewer metastases and cases with widespread bone metastases $(77 \%, 75 \%)$, but a significant difference $(\mathrm{P}=0.005)$ was observed in the rates of complete disappearance of pain in cases with 10 or fewer metastases (54\%) and widespread bone metastases (24\%) [6]. The reason for this may be that if the skeletal tumor burden is small, the accumulation of Sr-89 in each focus is higher and the pain improvement effect is greater than that in widespread bone metastases with the same amount of Sr-89 distributed over a large tumor burden. However, in our study, the relationship between BSI and pain relief owing to Sr-89 was not statically significant. We did not evaluate the rate of complete disappearance of pain as sample size is small and this study is retrospective study. However, although there are differences between prostate cancer and breast cancer, we cannot state that bone metastasis tumor burden is involved in the effects of Sr-89 on pain. Windsor et al. reported that in 75 prostate cancer bone metastasis patients, a superscan was observed in seven cases, with no pain relief in five [7]. According to the European Association of Nuclear Medicine guidelines, administration in cases of superscan is contraindicated [8]. Blake et al. reported that more than 5 times the amount of radiopharmaceutical is accumulated in cases of superscan than in single bone metastases [9]. Ackery et al. reported that no pain relief effect was demonstrated with $\leq 80 \%$ of the usual dose [10]. However, we observed pain relief in $5 / 8(62.5 \%)$ patients with $\mathrm{BSI}>3.028$ (median value) without more than Grade 3 toxicity.

McEwan et al. reported observing more pain improvement effect in the early stages of bone metastasis [11]. We found no statistically significant difference in 
pain improvement among cases involving different intervals between onsets of bone metastasis detected until Sr-89 administration. Moreover, zoledronic acid or denosumab is often used for patients with bone metastases. Yamada et al. reported that when zoledronate and Sr-89 were used simultaneously, the pain improvement effect was superior than when zoledronate or Sr-89 was used alone [12], suggesting an additive or synergistic effect. There are no reports on the concomitant use of denosumab and Sr-89. However, no significant difference was observed between Sr-89 with zoledronate acid and Sr-89 with denosumab.

In our study, Sr-89 was effective in $70 \%$ of cases; however, positive predictive factors for pain relief could not be determined. In addition, external beam radiation, BSI, and duration from bone metastasis diagnosis onset until Sr-89 administration were not predictive factors for pain relief.

This study had limitations. First, the sample size of the study was small. Second this study had a retrospective design. Therefore, further accumulation of patients and Ko

\section{Conclusion}

In patients with breast cancer bone metastases, $\mathrm{Sr}-89$ is effective in $70 \%$ of cases, although positive predictive factors for pain relief remained unknown.

\section{Acknowledgements}

We thank Kazuho Suyama of Nihon Koukan Hospital Director of Urology for help in this research.

\section{Conflicts of Interest}

The authors declare no conflicts of interest regarding the publication of this paper.

\section{References}

[1] Sanuki, N., Ono, A., Komatsu, E., Kamei, N., Akamine, S., Yamazaki, T., Mizunoe, S. and Maeda, T. (2012) Association of Computed Tomography-Detected Pulmonary Interstitial Changes with Severe Radiation Pneumonitis for Patients Treated with Thoracic Radiotherapy. Journal of Radiation Research, 53, 110-116. https://doi.org/10.1269/jrr.110142

[2] Nieder, C., Grosu, A.L., Andratschke, N.H. and Molls, N. (2005) Proposal of Human Spinal Cord Reirradiation Dose Based on Collection of Data from 40 Patients. International Journal of Radiation Oncology ${ }^{\star}$ Biology ${ }^{\star}$ Physics, 61, 851-855. https://doi.org/10.1016/j.ijrobp.2004.06.016

[3] Baziotis, N., Yakoumakis, E., Zissimopoulos, A., Geronicola-Trapali, X., Malamitsi, J. and Proukakis, C.H. (1998) Strontium-89 Chloride in the Treatment of Bone Metastases from Breast Cancer. Oncology, 55, 377-381.

https://doi.org/10.1159/000011881

[4] Fuster, D., Herranz, D., Vidal-Sicart, S., Munoz, M., Conill, C., Mateos, J.J., Martin, F. and Pons, F. (2000) Usefulness of Strontium-89 for Bone Pain Palliation in Metastatic Breast Cancer Patients. Nuclear Medicine Communications, 21, 623-626. https://doi.org/10.1097/00006231-200007000-00004 
[5] Dafermou, A., Colamussi, P., Giganti, M., Cittanti, C., Bestagno, M. and Piffanelli, A. (2001) A Multicentre Observational Study of Radionuclide Therapy in Patients with Painful Bone Metastases of Prostate Cancer. European Journal of Nuclear Medicine, 28, 788-798. https://doi.org/10.1007/s002590100533

[6] Kraeber-Bodéré, F., Campion, L., Rousseau, C., Bourdin, S., Chatal, J.F. and Resche, I. (2000) Treatment of Bone Metastases of Prostate Cancer with Strontium-89 Chloride: Efficacy in Relation to the Degree of Bone Involvement. European Journal of Nuclear Medicine, 27, 1487-1493. https://doi.org/10.1007/s002590000315

[7] Windsor, P.M. (2001) Predictors of Response to Strontium-89 (Metastron) in Skeletal Metastases from Prostate Cancer: Report of a Single Centre 10-Year Experience. Clinical Oncology, 13, 219-227. https://doi.org/10.1053/clon.2001.9257

[8] Guidelines for the Therapeutic Administration of Strontium 89. Radiaton Safety Program Department of Human Service. Publication No. 95/0386.6.

[9] Blake, G.M. and Fogelman, I. (2013) Bone: Strontium Ranelate Does Not Have an Anabolic Effect on Bone. Nature Reviews Endocrinology, 9, 696-697. https://doi.org/10.1038/nrendo.2013.210

[10] Ackery, D. and Yardley, J. (1993) Radionuclide-Targeted Therapy for the Management of Metastatic Bone Pain. Seminars in Oncology, 20, 27-31.

[11] McEwan, A.J. (2000) Use of Radionuclides for the Palliation of Bone Metastases. Seminars in Radiation Oncology, 10, 103-114. https://doi.org/10.1016/S1053-4296(00)80047-8

[12] Yamada, K., Yoshimura, M. and Kaise, H. (2012) Current Use of Sr 89 Chloride with Zoledronic Acid Is Safe and Effective for Breast Cancer Patients with Painful Bone Metastases. Experimental and Therapeutic Medicine, 3, 226-230. https://doi.org/10.3892/etm.2011.405 